\title{
LII. An interferometer method of determining the phase difference resulting from metallic reflexion
}

\section{H.P. Waran M.A.}

To cite this article: H.P. Waran M.A. (1922) LII. An interferometer method of determining the phase difference resulting from metallic reflexion, Philosophical Magazine Series 6, 43:255, 471-477, DOI: $10.1080 / 14786442208633898$

To link to this article: http://dx.doi.org/10.1080/14786442208633898

曲 Published online: 08 Apr 2009.

Submit your article to this journal $₫$

Q View related articles $₫$

4 Citing articles: 1 View citing articles 준 
The results agree sufficiently well to establish the existence of at least two periodic terms, but the differences between the various solutions are so large that it is not easy to say whether three or even a greater number may not be involved.

The somewhat unsatisfactory nature of this conclusion is solely due to the circumstance that the measured ordinates may be in error by an amount of the order of one-tenth of the maximum amplitude of swing, and as analyses of artificially constructed functions show, small changes in the values of the ordinates make unexpectedly large alterations in the values of speed and other constants.

Until me:uns are found for obtaining a higher degree of magnification of the movement, and, if possible, a diminution in the breadth of the trace, it will be difficult to obtain more definite results.

University of London,

King's College.

Aug. 1921 .

LII. An Interferometer Method of determining the Phase Difference resulting from Metalite Reftecion. $B y \mathrm{H} . \mathrm{P}$. WARAN, M.A.; Government Scholar of the Cniversity of Madras *

\section{[Plate VIII.]}

W HILE investigating the possibility of a parallel plate interferometer, by floating one transparent liquid as water over another like mercury, the question arose as to the difficulties that would arise from the difference in the character of the reflexions from the two surfaces. In the parailel plate of glass both the surfaces, are identical and the reflexions take place under identical conditions. But in the present case nearly total reflexion at the top water-iir surface and simple reflexion at the bottom mercury surface are utilised to get multiple reflexions from the parallel plate of liquid. To see if this metallic reflexion at the bottom surface was producing any disturbing factor prejudicial to the success of this type of interferometer, a control experiment was derised with a glass Lummer plate which led to a very interesting observation.

The glass Lummer plate (made by Hilger) was mounted on a specially made ebonite mount, resembling very much the

* Communicated by Prof. A. W. Porter, F.R.S. 


\section{Mr. H. P.Waran: Interferometer Method of determining}

usual brass mount, but provided with a trough arrangement as shown in fig. 1 in part section, by which clean mercury could be introduced below the plate and made to cover its bottom surface. This combination being of identical size with the usual mount of the parallel plate could be fitted on the Hilger interference spectroscope between the collimator and the constant deviation prism, as in fig. $a$ (Pl. VIII.).

Fig. 1.

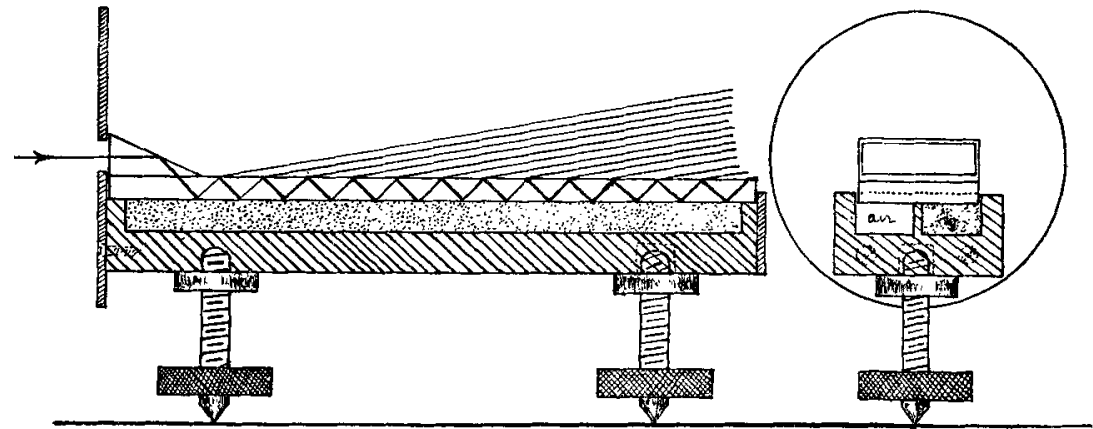

The slit was illuminated by a mercury vapour lamp, and the resulting interference pattern displayed an interesting change as shown by fig. $b$ (Pl. VIII.). For the first appearance it was as if an extra system of bands had erept in between the normal system of bands. That it was not due to a simple reflected system was evident from the absence of constant spacing between the two sets of bands. An idea that it might be due to a thin layer of air between the glass and the mercury led to a repetition of the experiment by silvering the bottom surface of the plate chemically. The same type of band system observed made it conclusive that it was being brought about as an effect of the metallic reflexion at the bottom surface.

Considering the effect of such a metallic reflexion, we know from the optical properties of metals that the emergent light will be elliptically polarized. On this consideration, the extra system of bands could be explained away directly as due to a simple phase difference effect brought about by the metallic reflexion. In such a case the effect of polarizing the incident. light ought to be to make one of these two systems of bands disappear as shown in fig. 2, according as the incident light is polarized in the plane of incidence or perpendicular to it. A Nicol polarizer introduced into the path of the incident light brings about this change as expected, confirming the validity of our explanation. 
Considering the accuracy with which interferometer measurements could be conducted, it occurred to the writer that this method may be used for experimentally determining the phase difference resulting from metallic reflexion with greater accuracy, since it is free from the defects of other methods which suffer * from the unavoidable impurities of the polished metal surfaces exposed to air.

Before going into the theory of the method, a word may be said about the experimental arrangements adopted to secure the necessary photographs of the fringes for measurement.

Fig. 2.- Showing the types of changes resulting from metallic reflexion in polarized and unpolarized light.

\begin{tabular}{|c|c|c|c|c|}
\hline \multicolumn{2}{|c|}{ Light not polarised } & \multicolumn{3}{|c|}{ Light polarised } \\
\hline $\begin{array}{c}\text { One of the } \\
\text { normal } \\
\text { band system } \\
\text { in air. }\end{array}$ & $\begin{array}{c}\text { After } \\
\text { metallic } \\
\text { reflection. }\end{array}$ & $\begin{array}{c}\text { In the plane } \\
\text { of } \\
\text { ineidence. }\end{array}$ & $\begin{array}{c}\text { At } 45^{\circ} \text { to } \\
\text { the plane } \\
\text { of } \\
\text { incidence. }\end{array}$ & $\begin{array}{c}\text { Perpendicular } \\
\text { to the plane } \\
\text { of } \\
\text { incidence. }\end{array}$ \\
\hline & & & & \\
\hline
\end{tabular}

The glass Lummer plate chemically silvered on the bottom surface was taken, and half the silvering along its length removed by gentle rubbing with dilute nitric acid, and the glass portion thus showing cleaned and dried carefully. It was then mounted in its usual stand on the Hilger interference spectroscope. Ijight from a mercury vapour lamp was focussed on the slit by a pair of collimating lenses, between which was interposed a 2 in. Nicol polarizer as illustrated in fig. $c$ (Pl. VIII.). The polarizer was set to polarize the incident light in the plane of incidence, and thus cut off the other component set of bands resulting from the metallic reflexion and corresponding to the case of light polarized perpendicular to the plane of incidence. Under these circumstances, the collimated beam in passing through this half motal and half air plate was split into two halves, namely, the one that underwent metallic reflexion and the other that did not, and the fringes due to these two were obtained consecutively on the same plate, but with a natural

* Drude's 'Theory of Optics,' p. 366. 
$47 \pm$ Mr. H. P. Waran : Interferometer Method of determinin!

displacement vertically between the two sets that represented the phase difference. The fringes due to the latter acted as the reference marks from which to measure the distance to the bands corresponding to the former, and a slight lateral shift given to the latter system of bands by rotating the wave-length drum a little served to identify the two in practice, as shown in fig. $d$ (Pl. VIII.).

For mercury, which was the next metal studied, the arrangement had to be slightly modified, and the specially made ebonite trough described above and shown in fig. 1 and Pl. VIII. fig. e, had to be used. A modification had to be introduced in it in the form of a narrow partition to divide the mercury chamber into two longitudinal halves, so that, as with the silver, we might get half the plate covered with mercury, leaving the other half free as shown in part section fig. 1.

We may proceed now to consider the theory of the method and the process employed for the actual evaluation of the phase difference from a measurement of the displacement of the metal fringes from the normal system.

Let $t=$ the thickness of the parallel plate

and $\mu=$ the refractive index for the wave-length $\lambda$ used, which may be calculated out from the given optical constants of the plate, using Cauchy's formulæ.

Then the path difference between two adjacent beams emerging out of the plate because of the multiple reflexions is given by the relation

$$
2 \mu t \cos r=n \lambda
$$

where $r$ is the angle of incidence for these rays within the plate.

If we count this as the path difference for the starting band of the Lummer system of fringes, we have a bright band at every position corresponding to an increase in the path difference by $\lambda$. Thus for the $p$ th band we have

$$
2 \mu t \cos \left(r-\delta r_{p}\right)=(n+p) \lambda \text {. }
$$

Subtracting (1) from $\left(2\right.$; , we have, since $\delta r_{p}$ is small,

$$
2 \mu t \sin r^{\cdot} \delta r_{p}=p \lambda \text {. }
$$

Further, we have the relations

and

$$
\sin i=\mu \sin r
$$

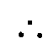

$$
\begin{aligned}
\sin i_{p} & =\mu \sin r_{p}, \\
\sin i-\sin i_{p} & =\mu\left(\sin r-\sin r_{p}\right)
\end{aligned}
$$

$$
=\mu \cdot 2 \cdot \cos \frac{r+r_{p}}{2} \sin \frac{r \cdot-r_{p}}{2} \text {. }
$$


But

$$
\frac{r+r_{p}}{2}=r \text { approx. and } \quad \sin ^{r-r_{p}}=\sin \frac{\delta r_{r}}{2}=\frac{\delta r_{p}}{2} \text {. }
$$

Therefore

$$
\sin i-\sin i_{p}=\mu \cos \gamma \delta r_{p} . \quad \text {. . . . }
$$

But from (3)

$$
\delta r_{p}=\frac{p \lambda}{2 \mu t \sin }
$$

substituting in (4) for $\delta r_{p}$ we get

$$
\begin{aligned}
\sin i-\sin i_{p} & =\frac{\mu \cos r p \lambda}{2 \mu^{t} \sin r} \\
& =\frac{p \lambda}{2 t \tan r} .
\end{aligned}
$$

Expanding

$$
2 \cos \frac{i+i_{p}}{2} \sin \frac{i-i_{p}}{2}=\underset{2 t \tan r}{p \lambda}=K_{p},
$$

where

$$
\mathrm{K}=\frac{\lambda}{\because t \frac{\tan r^{\circ}}{}} .
$$

Iuet

$$
i-i_{p}=\theta_{p} \text {, so that } i_{p}=i-\theta_{p} \text {, }
$$

and

$$
\frac{i+i_{p}}{2}=i-\frac{\theta_{p}}{2}
$$

and

$$
\sin \frac{i-i_{p}}{2}=\sin \frac{\theta_{p}}{2}=\frac{\theta_{p}}{2} .
$$

$\therefore$ We get

$$
\cos \left(i-\frac{\theta_{p}}{2}\right)=\frac{\mathrm{K}_{\underline{p}}}{\theta_{p}} \text {. . . . . . . }
$$

Now $\theta_{p}$ can be found by observation of the distance of the $p$ th band from the zero band and dividing it by the focal length of the camera lens. Substitating in equation (5) gives us the value of $i$, since $k$ is readily evaluated from the known constants. By taking every value of $p$ from 1 upwards, the corresponding value of $i$ is deduced and the mean value of $i$ found out. Values of $i$ for the successive bands can then be found out by calculation from the relation.

$$
\sin i_{p}=\sin i-\mathrm{K} p . . . \quad \text {. . . }
$$

After this preliminary consideration of the elementary theory applicable to the present case, we proceed as follows to apply it to the case of metallic reflexion. When simple multiple reflexion takes place in a parallel plate of glass bounded by air, we have the simple relation

$$
2 \mu t \cos r=n \lambda \text {. }
$$


476 Interferometer Method of determining Phase Iifference.

But, for the case of light reflected from one of the surfaces covered by metal and polarized in the plane of incidence, this becomes

$$
2 \mu t \cos (r+\delta r)+\frac{\theta}{2 \pi} \lambda=n \lambda . . .
$$

when we account for the displaced band as being due to a change of phase on metallic reflexion. When the band shifts to its new position as a result of the phase difference, the change in the angle of incidence corresponds to the term $\delta r$ and the phase difference of $\theta$ is taken into consideration by the term $\frac{\theta}{2 \pi} \lambda$.

Expanding (7),

$2 \mu t \cos r \cos \delta r-2 \mu t \sin r \sin \delta r+\frac{\theta}{2 \pi} \lambda=n \lambda$,

i.e. $2 \mu t \cos r-2 \mu t \sin r \delta r+\frac{\theta}{2 \pi} \lambda=n \lambda$,

i. e. $\quad n \lambda-2 \mu t \sin r \delta r+\frac{\theta}{2 \pi} \lambda=n \lambda$,

i. e. $\quad 2 \mu t \sin r \delta r=\frac{\theta}{2 \pi} \lambda$,

and therefore

$$
\theta=\frac{2 \pi}{\lambda} 2 \mu t \sin r \delta r . \quad . \quad .
$$

But

$$
\sin i=\mu \sin r
$$

Differentiating $\quad \cos i \delta i=\mu \cos r \delta r$.

Therefore

$$
\delta r=\frac{\cos i \delta i}{\mu \cos r}
$$

Substituting for $\delta r$ in (8)

$$
\begin{aligned}
\theta & =\frac{2 \pi}{\lambda}(2 \mu t \sin r) \frac{\cos i \delta i}{\mu \cos r} \\
& =4 \pi t \tan r \cos i \delta i \\
& =\mathrm{K}^{\prime} \cos i \delta i, \ldots .
\end{aligned}
$$

where

$$
\mathrm{K}^{\prime}=\frac{4 \pi}{\lambda} t \tan r
$$

In practice, with the constant $\mathrm{K}^{\prime}$ evaluated from the known constants of the plate, to evaluate $\theta$ the phase difference, the normal (glass-air) system of bands is measured out, and getting by subtraction the distance of each from 0 and 
dividing by the focal length of the camera, the values of $\theta_{p}$ and therefrom the mean $i, i_{p}$, and $\cos i_{p}$ for each value of $p$. are evaluated. In each case $\delta i_{p}$ is given by the measured displacement on the plate of the metallic fringe from the normal system divided by the focal length of the camera lens. The mean of the values of $\cos i_{p} \delta i_{p}$ for each of the values of $p$ is the value for cos $i \delta i$ employed in the final substitution in (9) to give the value of the phase difference.

In practice, the method works out quite satisfactorily, and the preliminary stady in the case of silver and mercury has yielded quite concordant results. Because of freedom from surface impurities in the present method, and considering that the effect of surface impurities is to lower the values, the slightly higher values obtained by this method are easily explained. This view has also in corroboration the fact of the observed difference in the case of mercury being much smaller than with silver, which is more liable to surface contamination than mercury. Further, the method has also the advantage of being applicable to all the other metals which can be coated on to the Interferometer plate by cathodic deposition or otherwise. A detailed evaluation of this phase difference for all the metals is in progress.

In conclusion, I beg to express my indebtedness to Prof. R. Ll. Jones, M.A., Professor of Physics, Presidency College, Madras, for his kind and sympathetic help during the progress. this investigation at Madras under his direction.

University College, London.

LIII. Stopping Power and Atomic Number. By J. L. Glasson, M.A., D.Se.*

1. T $\mathrm{T}$ has recently been established that certain properties of the atom are more simply and accurately expressed in terms of atomic number than in terms of atomic weight. The following is an attempt to find out if this is also true of the atomic property known as stopping power for a rays.

According to the work of Sir William Bragg, it was shown. that the stopping power of an atom is proportional to the square root of the weight of the atom. Professor Braggs own figures which form the basis of the work are given in.

* Communicated by Prof. Sir E. Rutherford, F.R.S. 
WARAN.

Phil. Mag. Ser. 6, Vol. 43. Pl. VIII.

(a)

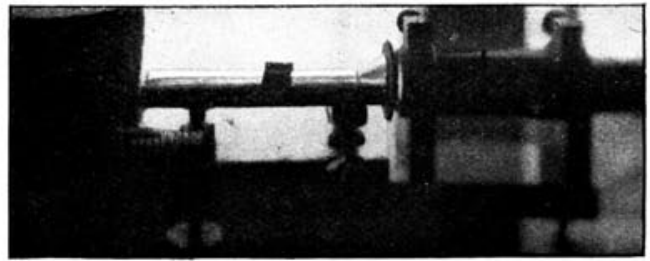

(d)

(c)

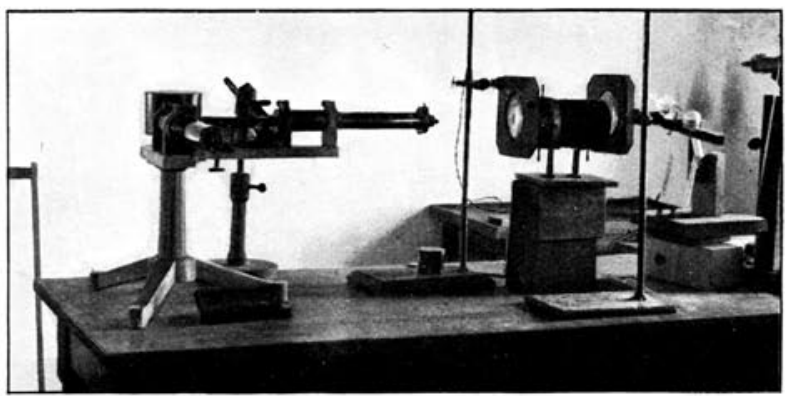

(e)

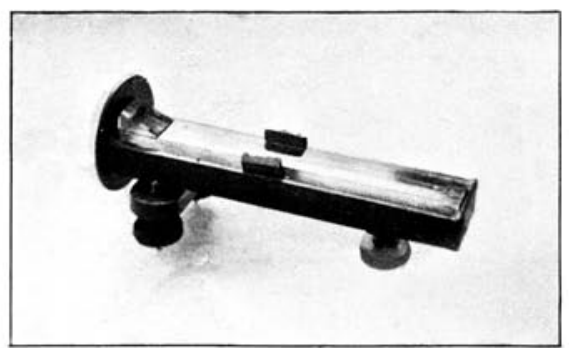

